\title{
Bone metastases in gastric cancer follow a RANKL-independent mechanism
}

\author{
LUCIA D'AMICO ${ }^{1 *}$, MARIA ANTONIETTA SATOLLI ${ }^{2 *}$, CATERINA MECCA $^{2}$, \\ ANNA CASTIGLIONE ${ }^{3}$, MANUELA CECCARELLI ${ }^{3}$, PATRIZIA D'AMELIO ${ }^{4}$, MAURO GARINO $^{5}$, \\ MAURIZIO DE GIULI ${ }^{5}$, SERGIO SANDRUCCI ${ }^{6}$, RICCARDO FERRACINI $^{7}$ and ILARIA ROATO ${ }^{1}$ \\ ${ }^{1}$ CeRMS (Center for Experimental Research and Medical Studies), San Giovanni Battista Hospital; \\ ${ }^{2}$ Department of Medical Oncology, San Giovanni Battista Hospital; ${ }^{3}$ Unit of Cancer Epidemiology, \\ San Giovanni Battista Hospital; ${ }^{4}$ Department of Surgical and Medical Disciplines, University of Turin; \\ ${ }^{5}$ Department of Surgery, San Giovanni Battista Hospital and University of Turin; Departments of \\ ${ }^{6}$ Oncologic Surgery and ${ }^{7}$ Orthopaedics, San Giovanni Battista Hospital, Turin, Italy
}

Received November 22, 2012; Accepted December 27, 2012

DOI: $10.3892 / o r .2013 .2280$

\begin{abstract}
Gastric cancer is one of the most common and lethal malignancies worldwide. Bone metastases in gastric cancer are less common than in other solid tumors, but when they occur the prognosis is generally poor. Increased osteoclastogenesis and osteoclast activity are common features in bone metastases caused by different osteotropic cancer. We investigated osteoclastogenesis and its mechanisms in gastric cancer by enrolling 31 newly diagnosed gastric cancer patients and 45 healthy controls. We studied in vitro osteoclastogenesis in the peripheral blood mononuclear cell cultures of patients and controls, showing spontaneous osteoclastogenesis for half of the patients. This osteoclastogenesis was RANKL- and TNF- $\alpha$-independent. We analyzed primary tumor and bone metastatic tissues of gastric cancer for the expression of genes involved in osteoclastogenesis. The expression of transforming growth factor- $\beta$ (TGF- $\beta$ ), osteoprotegerin (OPG), IL-7 and dickkopf-1 (DKK-1) was higher in primary tumors than in bone metastases. RANKL was not detectable in primary tumor or in bone metastatic tissue. The serum RANKL level was significantly higher in healthy controls than in patients, and it was not related to osteoclastogenesis, thereby suggesting that RANKL is not involved in the bone metastatic mechanisms in gastric cancer. We hypothesized a role of RANKL in angiogenesis, thus we compared the serum levels of RANKL to those of VEGF, since VEGF is directly related to angiogenesis. Different from RANKL, the VEGF serum levels were higher in gastric patients than in controls, suggesting a block
\end{abstract}

Correspondence to: Dr Ilaria Roato, CeRMS, San Giovanni Battista Hospital, via Santena 5, I-10126 Turin, Italy

E-mail: roato78@libero.it

*Contributed equally

Key words: gastric cancer, bone metastasis, osteoclast, RANKL of the angiogenesis inhibition due to RANKL. RANKL and VEGF serum levels were not predictive of overall survival in our cohort of gastric patients.

\section{Introduction}

In spite of the marked spontaneous decline in the incidence of stomach cancer in most Western countries, in Asia it remains the second most common type of cancer, following lung cancer, and accounts for $13 \%$ of all tumors (1-3). In Italy, the 5-year relative survival rate is approximately $30 \%$ (4). Although surgical techniques have improved, only a small percentage of patients are diagnosed with a localized disease amenable to surgery (5). The outcome of patients with clinically advanced disease is usually poor although new chemotherapeutic regimens are available. Bone metastases in gastric cancer are rare and they usually appear late in the natural history of the disease; however, micrometastatic seeding in the bone may be evident in the early stage of the disease (5). Nevertheless, when these metastatic lesions occur, the prognosis is poor, as they can cause bone marrow suppression, anemia, cord compression and intravascular coagulation and can quickly lead to mortality (6). In early gastric cancer, a poorly differentiated carcinoma and the presence of signet-cells seem to be associated with bone metastases (7). The mechanism of bone metastases in gastric cancer remains to be clarified, since there are currently different hypotheses concerning the metastatic spread to the bone, such as through portal vein, lymphatic channel (7) and vertebral vein system (8). In general, gastric cancer bone metastases result from a diffuse metastatic spread in the bone marrow, most frequently localized in the thoracic and lumbar vertebrae (9), and they are osteolytic or, less commonly, mixed osteolytic/osteoblastic.

In osteotropic tumors such as breast, prostate and lung cancer, osteoclasts (OCs) are mainly responsible for bone destruction in patients with metastatic bone disease. Several molecules have been identified as the mediators of bone metastases in solid tumors, for example the RANK/RANKL/ 
osteoprotegerin (OPG), TNF- $\alpha$ and PTHrP system (10-12). In addition to the known role of RANKL in the skeletal and immune systems (13), recent studies also reported a role of RANKL in angiogenesis. Certain authors support an inhibitory role of RANKL $(14,15)$, whereas others showed a stimulating action on angiogenesis (16). Contrary to RANKL, VEGF is known as a potent mitogen angiogenic factor and is one of the most important molecules involved in the vascularization of bone tissue (17), thus its serum levels are considered an index of angiogenesis. Moreover, increased VEGF serum levels are associated with a poor clinical outcome in gastric cancer (18).

In this study, we examined the mechanisms of bone metastases by gastric cancer investigating the presence of spontaneous osteoclastogenesis in vitro, the expression of genes involved in bone metastasis by gastric tumor tissues and any correlations among the serum levels of RANKL, VEGF and clinical parameters in our cohort of patients.

\section{Materials and methods}

Patients. Between October 2008 and February 2011, we collected blood samples and sera from 31 consecutive newly diagnosed gastric cancer patients at the San Giovanni Battista Hospital in Turin. We also collected samples from 45 healthy controls. The study design was approved by the Ethics Committee of the hospital. Clinicopathological data for all patients were collected in a database.

Cell cultures. Peripheral blood (PB) samples were obtained from 16 gastric cancer patients and 19 healthy controls, processed as previously described (19). Briefly, peripheral blood mononuclear cells (PBMCs) were isolated following centrifugation over a density gradient, according to the Ficoll method. The number of PBMCs retrieved from the blood samples varied among patients and controls, thus it was not possible to perform all the experimental procedures on all the enrolled patients and controls. PBMCs were plated in 24 -well plates, using $\alpha$-minimal essential medium ( $\alpha$-MEM; Invitrogen, Carlsbad, CA, USA), supplemented with $10 \%$ fetal bovine serum, benzylpenicillin (100 IU/ml) and streptomycin $(100 \mathrm{mg} / \mathrm{ml}$; Lonza, Basel, Switzerland) and maintained at $37^{\circ} \mathrm{C}$ in a humidified atmosphere of $5 \% \mathrm{CO}_{2}$. To obtain fully differentiated human OCs, PBMCs were cultured in the presence or absence of recombinant human M-CSF $(25 \mathrm{ng} / \mathrm{ml})$ and RANKL (30 ng/ml), for 15 days. In 8 independent experiments, PBMCs were cultured in the presence of increasing concentrations of RANKFc (30-50 ng/ml) and anti-TNF- $\alpha$ (1.5-3-5 mg/ml; PeproTech, London, UK).

At the end of the culture period, cells were stained for tartrate-resistant acid phosphatase (TRAP, kit supplied by Sigma-Aldrich, St. Louis, MO, USA) and OCs were identified as TRAP positive, multinucleated cells, containing three or more nuclei.

Assay of bone resorption activity. To study OC resorbing activity, PBMCs from patients and healthy controls were plated on a BioCoat osteologic bone cell culture system provided by BD Biosciences (Bedford, MA, USA) and cultured for 20 days. In order to visualize pits formed by OCs, the cells were removed by washing each well with $\mathrm{NaOCl}$ and resorption lacunae were identified by light microscopy. The quantification of resorbing area was performed by a semi-automated image analyzing system (20).

Real-time quantitative analysis of TGF- $\beta, O P G, D K K-1, S O S T$, RANKL and IL-7 gene expression. Total RNA was extracted by the TRIzol system (Invitrogen) from surgically resected primary tumor and bone biopsies from patients previously diagnosed with bone metastasis by gastric cancer. The first-strand cDNA synthesis was performed as previously described (21).

Quantitative analysis of transforming growth factor- $\beta$ (TGF- $\beta$ ), osteoprotegerin (OPG), dickkopf-1 (DKK-1), sclerostin (SOST), RANKL and IL-7 were performed with real-time quantitative PCR (RQ-PCR) using $\beta$-actin as the housekeeping control. RT-PCR was carried out using the iCycler $\mathrm{iQ}^{\mathrm{TM}}$ system (Bio-Rad, Hercules, CA, USA). TaqMan probes were designed using Primer Express v2.0 software and synthesized by Applied Biosystems. The sequences of the probes and primers were previously described (22). All the probes were labeled at the 5'-end with 6-carboxyfluorescein (FAM) and the 3'-end with 6-carboxytetramethylrhodamine (TAMRA). Reactions for gene quantification were performed in a $25-\mu 1$ final volume with $2 \mu \mathrm{l}$ of sample cDNA, $1 \mathrm{X}$ iQ Supermix (Bio-Rad), $0.3 \mu \mathrm{M}$ of each primer and $0.4 \mu \mathrm{M}$ of the probes. PCR primers were the same as those used for gene cloning. The amplification conditions for quantization were: $95^{\circ} \mathrm{C}$ for $15 \mathrm{~min}, 50$ cycles at $95^{\circ} \mathrm{C}$ for $15 \mathrm{sec}, 58^{\circ} \mathrm{C}$ for $1 \mathrm{~min}$.

ELISA. The serum levels of total RANKL (Biomedica Medizinprodukte GmbH and Co. KG, Vienna, Austria), IL-7 (BenderMed Systems, Vienna, Austria) and VEGF (R\&D Systems, Abingdon, UK) were determined by a commercially available ELISA kit according to manufacturer's instructions in all patients and healthy controls. Samples were assayed in duplicate and data were expressed as mean values.

Statistical analysis. The characteristics of the patients were described using medians and range for the continuous variables and using percentage frequencies for the categorical variables. We performed the Mann-Whitney test to assess difference of number of culture between patients and controls, difference of gene expression between primary tumor and bone biopsies, and difference of RANKL and VEGF between patients and healthy controls. Crude survival probabilities were estimated with the Kaplan-Meier method. The survival time was months since cancer diagnosis. A Cox proportional hazard model was employed to estimate the crude and adjusted hazard ratios (HRs) and 95\% confidence intervals. Analyses were performed using Stata 11.2.

\section{Results}

Patients. Among 31 patients newly diagnosed with gastric cancer, 16 patients had a localized disease and were subjected to surgery and 15 had a metastatic disease. The main clinical characteristics of the patients are summarized in Table I.

Evaluation of spontaneous osteoclastogenesis in vitro and OC activity. OCs spontaneously differentiated in vitro without the addition of M-CSF and RANKL (spontaneous osteoclasto- 
Table I. Patient baseline characteristics by metastatic status.

\begin{tabular}{|c|c|c|c|c|c|c|}
\hline & \multicolumn{2}{|c|}{$\begin{array}{c}\text { Non- } \\
\text { metastatic }\end{array}$} & \multicolumn{2}{|c|}{ Metastatic } & \multicolumn{2}{|c|}{ Total } \\
\hline & No. & $\%$ & No. & $\%$ & No. & $\%$ \\
\hline \multicolumn{7}{|l|}{ Gender } \\
\hline Female & 4 & 25 & 6 & 40 & 10 & 32.26 \\
\hline Male & 12 & 75 & 9 & 60 & 21 & 67.74 \\
\hline \multicolumn{7}{|l|}{ Age } \\
\hline$<60$ & 7 & 43.75 & 9 & 60 & 16 & 51.61 \\
\hline$\geq 60$ & 9 & 56.25 & 6 & 40 & 15 & 48.39 \\
\hline
\end{tabular}

Tumor site

$\begin{array}{lrrrrrr}\text { Stomach } & 16 & 100 & 14 & 93.33 & 30 & 96.77 \\ \text { Cardias } & 0 & 0 & 1 & 6.67 & 1 & 3.23\end{array}$

Grading

1

2

3

Not evaluated

$\begin{array}{rcrcrr}1 & 6.25 & 0 & 0 & 1 & 3.23 \\ 2 & 12.5 & 0 & 0 & 2 & 6.45 \\ 12 & 75.0 & 10 & 66.67 & 22 & 70.97 \\ 1 & 6.25 & 5 & 33.33 & 6 & 19.35\end{array}$

Histologic type

$\begin{array}{llcccrr}\text { Diffuse } & 7 & 43.75 & 8 & 53.33 & 15 & 48.39 \\ \text { Enteric } & 6 & 37.5 & 1 & 6.67 & 7 & 22.58 \\ \text { Mixed } & 1 & 6.25 & 1 & 6.67 & 2 & 6.45 \\ \text { Adenocarcinoma } & 2 & 12.5 & 5 & 33.33 & 7 & 22.58\end{array}$

Vascular invasion

Yes

$\begin{array}{llllll}7 & 43.75 & 3 & 20 & 10 & 32.26\end{array}$

No

$\begin{array}{llllll}9 & 56.25 & 12 & 80 & 21 & 67.74\end{array}$

Chemotherapy

$\begin{array}{lrrrrrr}\text { In } & 0 & 0 & 3 & 20 & 3 & 9.68 \\ \text { Out } & 16 & 100 & 12 & 80 & 28 & 90.32\end{array}$

Lymph node invasion

\begin{tabular}{crrrrrrr} 
N0 & 7 & 43.75 & & & & \\
N1 & 3 & 18.75 & & & & \\
N2 & 3 & 18.75 & & & & \\
N3 & 3 & 18.75 & & & & \\
Total & 16 & 100 & 15 & 100 & 31 & 100 \\
\hline
\end{tabular}

genesis) in half of the analyzed patients (8/16 cases), whereas with PBMC derived from the other 8 patients, OCs only originated after the addition of exogenous factor (Figs. 1 and 2). Gastric patients showed an increase of spontaneous osteoclastogenesis compared to healthy controls, but it was not statistically significant ( $\mathrm{p}=0.11)$ (Fig. 1).

The analysis of OC activity revealed that it did not increase in patients compared to controls; indeed, we did not observe significant variation in the percentage of resorption (data not shown). Since osteoclastogenesis is commonly dependent on RANKL and TNF- $\alpha$, we tested the effect of an anti-TNF- $\alpha$ antibody and RANK-Fc in PBMC cultures, showing that osteoclastogenesis was not inhibited, thus it was not dependent on these two factors (Fig. 3).

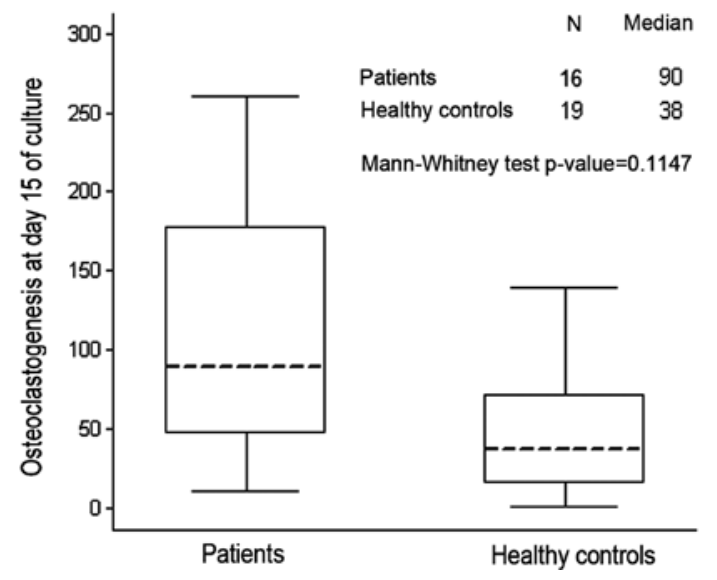

Figure 1. Spontaneous osteoclastogenesis in vitro. Comparison of osteoclastogenesis at day 15 between gastric tumor patients and healthy controls. Box plot displaying the outliers, the extremes, the upper and lower quartile and the median.
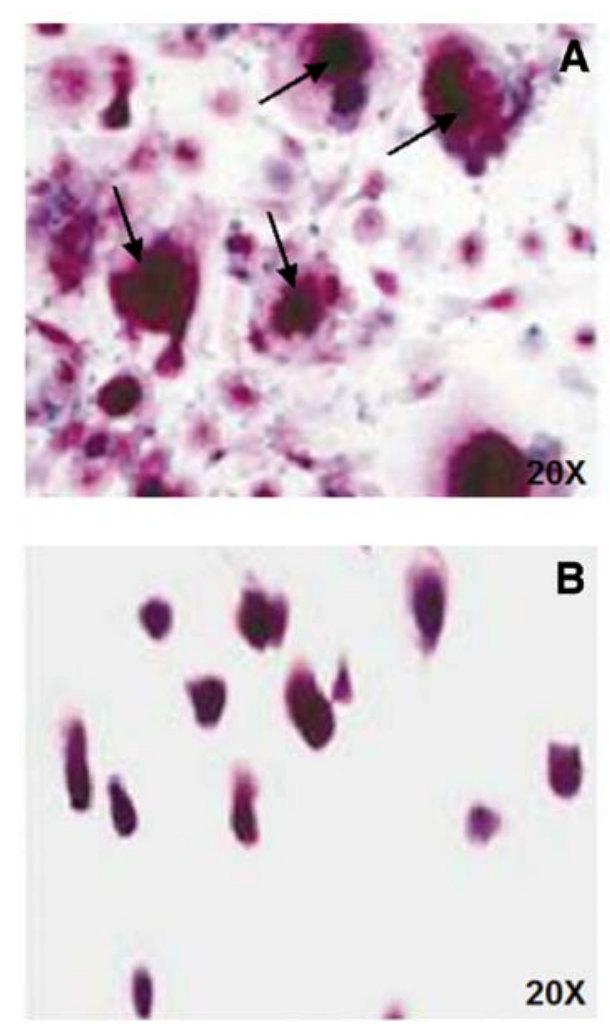

Figure 2. TRAP positive osteoclasts (OCs). (A) An example of TRAP positive OCs (arrows) derived from one of the 8 gastric patients showing spontaneous osteoclastogenesis. (B) No osteoclastogenesis or TRAP positive multinucleated OCs were detected in 8 patients. Magnification, x20.

Expression analysis of genes involved in osteoclastogenesis. In order to investigate whether gastric cancer cells expressed genes which regulate osteoclastogenesis, we analyzed the expression of TGF- $\beta$, RANKL, OPG, DKK-1, SOST and IL-7 in primary and bone metastatic gastric cancer tissues. TGF- $\beta$, OPG and DKK-1 were higher in the primary tumors than in the bone metastatic lesions. SOST was only expressed in the bone metastases, while IL-7 was only expressed in primary tumors (Table II). RANKL was not detectable in either tissue. 
Table II. Expression analysis of genes involved in osteoclastogenesis.

\begin{tabular}{|c|c|c|c|c|c|}
\hline & \multicolumn{2}{|c|}{ Primary tumor } & \multicolumn{2}{|c|}{ Bone metastases } & \multirow[b]{2}{*}{ p-value } \\
\hline & $\begin{array}{c}\text { Median } \\
\text { Copy N. genes/ } \beta \text {-actin }\end{array}$ & Range & $\begin{array}{c}\text { Median } \\
\text { Copy N. genes/ } \beta \text {-actin }\end{array}$ & Range & \\
\hline TGF- $\beta / \beta$-actin & 0.0154 & $0.0034-0.0236$ & 0.0030 & $0.0015-0.0045$ & 0.01 \\
\hline OPG/ $\beta$-actin & 0.0083 & $0.0027-0.3508$ & 0.0010 & $0.0005-0.0019$ & 0.46 \\
\hline DKK- $1 / \beta$-actin & 0.0004 & $0.0002-0.016$ & 0.0001 & $0-0.0002$ & 0.39 \\
\hline SOST $/ \beta$-actin & Not detected & & 0.0006 & $0.0003-0.003$ & \\
\hline IL-7/ß-actin & 0.0954 & $0.0507-1.6417$ & Not detected & & \\
\hline
\end{tabular}

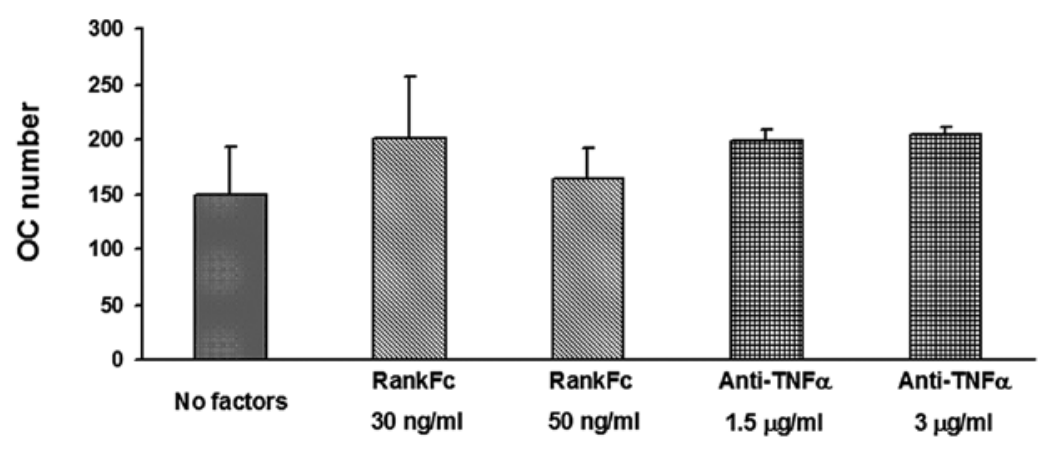

Figure 3. Spontaneous osteoclastogenesis is independent of RANKL and TNF- $\alpha$. The RANKFc and anti-TNF- $\alpha$ addition to unstimulated PBMC culture from gastric patients did not inhibit osteoclastogenesis.

A

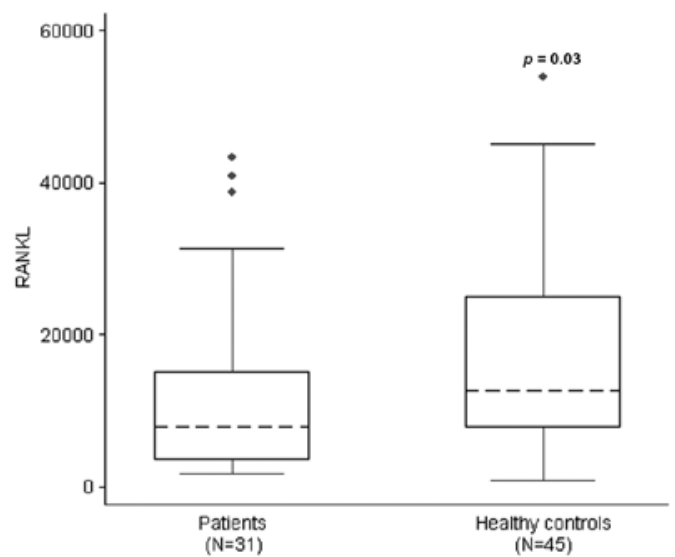

B

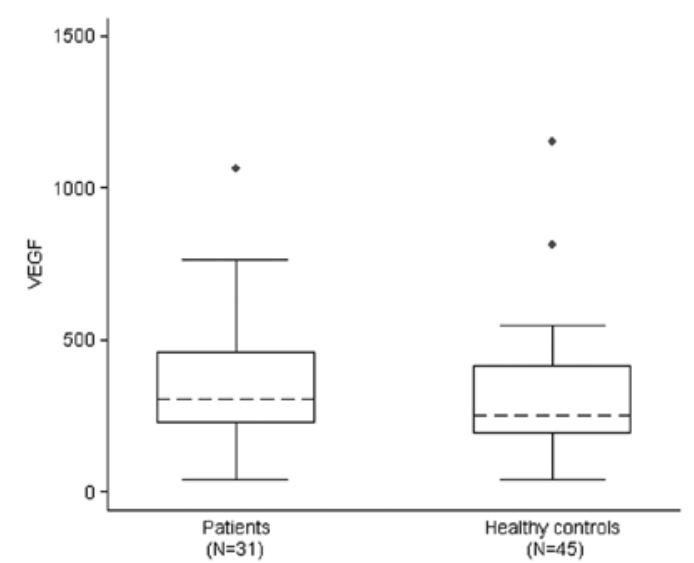

Figure 4. Serum levels of RANKL and VEGF. Comparison of RANKL (A) and VEGF (B) levels between patients with gastric cancer and healthy controls. Box plot displaying the outliers, the extremes, the upper and lower quartile and the median.

Serum level of IL-7, RANKL and VEGF. In previous studies on bone metastases by solid tumors, we demonstrated a role of IL-7 in promoting osteoclastogenesis (23). Thus, we administered IL-7 serum levels in 31 gastric cancer patients. Unlike other osteotropic tumors, such as lung cancer, in gastric patients IL-7 levels were not significantly different between patients with and without spontaneous osteoclastogenesis (the median values were 10.1 and $12.8 \mathrm{pg} / \mathrm{ml}$, respectively).

Serum RANKL levels were significantly higher in healthy controls than in patients (median, 12635.40 and $7772.80 \mathrm{pg} / \mathrm{ml}$; range, 818.7-53898.50 and 1643.00-43245.40 pg/ml, respectively; $\mathrm{p}=0.03$ ) (Fig. 4A). We did not observe any correlation between serum RANKL and the presence of spontaneous osteoclastogenesis, confirming the RANKL-indipendence of the detected osteoclastogenesis in vitro. The serum VEGF level was higher in patients than in healthy controls, but the difference was not statistically significant (median, 304 and $252 \mathrm{pg} / \mathrm{ml}$; range, 38-1064 and 39-1151 pg/ml, respectively; $\mathrm{p}=0.14)$ (Fig. 4B).

Analysis of prognostic value of RANKL and VEGF. In order to evaluate the prognostic value of RANKL and VEGF, we calculated the overall survival (OS) of 31 patients who were diagnosed with gastric cancer when the blood sample was 


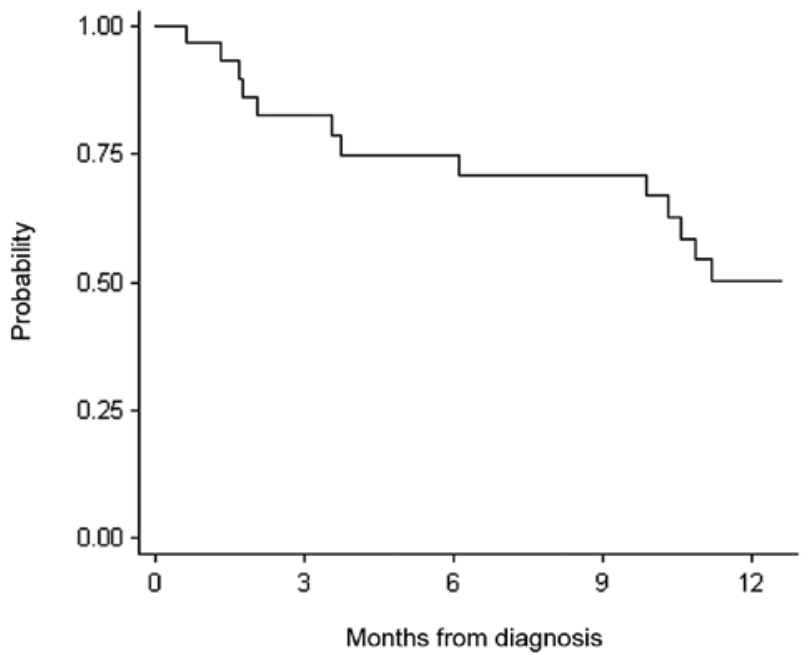

Figure 5. Overall survival of 31 gastric cancer patients. Overall survival at 12 months was 0.50 (95\% IC, $0.30-0.67$ ).

collected. As there is a lack of information regarding RANKL and VEGF values, the median value of serum RANKL and VEGF was chosen as cut-off for the analyses. OS at 12 months was 0.50 (95\% IC, 0.30-0.67) (Fig. 5). On multivariable Cox analysis, RANKL and VEGF (adjusted for metastatic status) were not predictive of OS (RANKL, HR, 1.10; 95\% IC, 0.38-3.19; VEGF, HR, 1.19; 95\% IC, 0.41-3.44). These results were also confirmed when adjusted for age and using RANKL and VEGF as continuous variables.

\section{Discussion}

One of the main characteristics of osteolytic bone metastasis is the increase of OCs and their activity. We studied the spontaneous osteoclastogenesis in in vitro cultures of PBMCs derived from gastric cancer patients. Spontaneous osteoclastogenesis was observed in half of the patients. Moreover, unlike other osteotropic solid tumors such as breast and lung cancer $(19,24,25)$, in gastric cancer the osteoclastogenesis was independent of RANKL and TNF- $\alpha$. We consider that other molecules could be involved in the stimulation of osteoclastogenesis, such as IL-11, which has been reported to support human osteoclastogenesis with IL-6 by a RANKLindependent mechanism (26). Moreover, IL-11 is key in gastric damage, mucosal repair and gastric cancer progression $(27,28)$.

Since the detected osteoclastogenesis did not correlate with the presence of bone metastases, we suggested an alternative hypothesis to explain it, considering a potential relationship between an increased osteoclastogenesis and the gastric acid levels, which are known to affect OC activity (29). We observed that some patients with increased osteoclastogenesis also showed chronic gastritis and referred to taking proton-pump inhibitors (data not shown). Although this requires further investigation, it might be possible that hypochlorhydria induced by drugs is responsible for the stimulation of $\mathrm{OC}$ resorption activity, leading to an increase of osteoclastogenesis in vitro.

To elucidate the production of molecules stimulating osteoclastogenesis by gastric cancer cells, we analyzed the mRNA expression on primary tumors and bone metastases of gastric cancer. TGF- $\beta$ was higher in primary tumor than in bone metastatic samples. TGF- $\beta$ is released in active form by bone matrix, upon tumor-induced osteoclastic bone resorption. It stimulates bone metastatic cells to secrete factors that further drive osteolytic destruction of the bone adjacent to the tumor (30). Our result is in accordance with a previous study, reporting an increased TGF- $\beta$ expression in gastric cancer, which is closely related to invasion and metastasis (31).

IL-7 was mainly expressed in the tumor tissue of gastric cancer patients, but its mean serum level was not significantly different between patients with or without osteoclastogenesis. This result differs from our previously published data on osteotropic tumors, where we demonstrated that IL-7 was high in patients with spontaneous osteoclastogenesis, as it stimulated OC differentiation through RANKL (23). In the present study, in gastric cancer, we did not show the same mechanism since RANKL did not promote osteoclastogenesis. Furthermore, we did not detect RANKL expression in primary tumor or in bone metastatic samples, and RANKL serum levels did not correlate with the presence of bone metastases. In particular, serum RANKL values were lower in patients than in controls, confirming our finding on RANKL-independent osteoclastogenesis. OPG was expressed in primary tumor and bone metastatic samples, according to a previous report that OPG provides a survival advantage to cancer cells (32). OPG correlates with aggressiveness and poor prognosis of gastric carcinoma (33). We did not observe any correlation between OPG expression and osteoclastogenesis, likely due to the RANKL-independence of osteoclastogenesis in our cohort of patients.

DKK-1 and SOST are soluble inhibitors of canonical WNT signaling (34), which plays an important role in bone development by inhibiting OC differentiation (35), stimulating osteoblastogenesis and mineralizing activity of osteoblasts (36). We detected DKK-1 expression in primary tumor, according to previously published data reporting its expression in some human specimens of tumors, and suggesting that a cancermediated modulation of WNT activity affects the metastatic phenotype (37-39). SOST was only detected in bone metastatic samples, according to the specific expression of these molecules by bone cells (34).

Since the collected data did not link RANKL to the bone metastasis pathogenesis, we investigated the potential involvement of RANKL in the tumor-induced angiogenesis. Previous data reported an inhibitory role of RANKL in angiogenesis $(14,15)$, which could explain its lower serum levels in patients compared to controls. To evaluate angiogenesis, we administered serum VEGF, which is a key angiogenic factor mediating neo-vascularization and could act as a surrogate marker of tumor angiogenesis (40). Unlike RANKL, the VEGF serum levels were higher in gastric patients than in controls, suggesting a block of the angiogenesis inhibition due to RANKL. We also evaluated the prognostic value of serum RANKL and VEGF, as in gastric cancer VEGF has been described as an independent prognostic factor of survival $(18,41)$. In our cohort of patients neither RANKL nor VEGF showed significant differences in the OS.

To our knowledge, this is the first study to investigate osteoclastogenesis and to evaluate the role of RANKL in gastric cancer. Although this study has certain limitations with regard to the single-centre design of this study and its small sample 
size, we aim to open new perspectives in the investigation of the molecular mechanisms involved in this process.

In conclusion, our results suggest that the common parameters and molecules regulating bone metastases in osteotropic solid tumors, such as breast, lung and prostate cancer, do not appear to be involved in the pathogenesis of bone metastases from gastric cancer. Indeed, neither spontaneous osteoclastogenesis in vitro nor serum RANKL concentrations correlated with bone metastases. RANKL could play a role in the angiogenesis of gastric cancer, however further investigations to define its pathophysiological role in gastric carcinoma are warranted.

\section{References}

1. Sasako M, Inoue M, Lin JT, Khor C, Yang HK and Ohtsu A: Gastric Cancer Working Group Report. Jpn J Clin Oncol 40 (Suppl 1): i28-i37, 2010.

2. Jemal A, Bray F, Center MM, Ferlay J, Ward E and Forman D: Global cancer statistics. CA Cancer J Clin 61: 69-90, 2011.

3. Yang L: Incidence and mortality of gastric cancer in China. World J Gastroenterol 12: 17-20, 2006.

4. AIRTUM Working Group: Italian cancer figures, report 2011 survival of cancer patients in Italy. Epidemiol Prev 35 (5-6 Suppl 3): 1-200, 2011 (In Italian).

5. Amadori D, Cascinu S, Conte PF and Ibrahim T: Bone metastases. In: Osteo-Oncology Textbook. Poletto Editore, Grafiche Cisalpina, S. Giuliano Milanese, pp200-201, 2010.

6. Kim HS, Yi SY, Jun HJ, et al: Clinical outcome of gastric cancer patients with bone marrow metastases. Oncology 73: 192-197, 2007.

7. Kobayashi M, Okabayashi T, Sano T and Araki K: Metastatic bone cancer as a recurrence of early gastric cancer - characteristics and possible mechanisms. World J Gastroenterol 11: 5587-5591, 2005.

8. Batson OV: The function of the vertebral veins and their role in the spread of metastases. 1940. Clin Orthop Relat Res (312): 4-9, 1995.

9. Devkaran B, Jhobta R and Verma DK: Bony metastasis of gastric adenocarcinoma. Saudi J Gastroenterol 15: 137-138, 2009.

10. Boyle WJ, Simonet WS and Lacey DL: Osteoclast differentiation and activation. Nature 423: 337-342, 2003.

11. Hofbauer LC, Neubauer A and Heufelder AE: Receptor activator of nuclear factor-kappaB ligand and osteoprotegerin: potential implications for the pathogenesis and treatment of malignant bone diseases. Cancer 92: 460-470, 2001

12. Kobayashi K, Takahashi N, Jimi E, et al: Tumor necrosis factor alpha stimulates osteoclast differentiation by a mechanism independent of the ODF/RANKL-RANK interaction. J Exp Med 191: 275-286, 2000

13. Walsh MC and Choi Y: Biology of the TRANCE axis. Cytokine Growth Factor Rev 14: 251-263, 2003.

14. McGonigle JS, Giachelli CM and Scatena M: Osteoprotegerin and RANKL differentially regulate angiogenesis and endothelial cell function. Angiogenesis 12: 35-46, 2009.

15. Sfiridaki K, Pappa CA, Tsirakis G, et al: Angiogenesis-related cytokines, RANKL, and osteoprotegerin in multiple myeloma patients in relation to clinical features and response to treatment. Mediators Inflamm 2011: 867576, 2011.

16. Min JK, Cho YL, Choi JH, et al: Receptor activator of nuclear factor (NF)-kappaB ligand (RANKL) increases vascular permeability: impaired permeability and angiogenesis in eNOSdeficient mice. Blood 109: 1495-1502, 2007.

17. Carlevaro MF, Cermelli S, Cancedda R and Descalzi Cancedda F Vascular endothelial growth factor (VEGF) in cartilage neovascularization and chondrocyte differentiation: auto-paracrine role during endochondral bone formation. J Cell Sci 113: 59-69, 2000

18. Vidal O, Metges JP, Elizalde I, et al: High preoperative serum vascular endothelial growth factor levels predict poor clinical outcome after curative resection of gastric cancer. Br J Surg 96 : $1443-1451,2009$
19. Roato I, Grano M, Brunetti G, et al: Mechanisms of spontaneous osteoclastogenesis in cancer with bone involvement. FASEB J 19: 228-230, 2005.

20. Brianza S, D'Amelio P, Cerrato M, Bignardi C, Grimaldi A, Pescarmona $\mathrm{G}$ and Isaia $\mathrm{G}$ : A dedicated image analysis software tool for the evaluation of the resorption activity of cultured osteoclasts. JIST 52: 30508-1-30508-9, 2008.

21. Roato I, D'Amelio P, Gorassini E, et al: Osteoclasts are active in bone forming metastases of prostate cancer patients. PLoS One 3: e3627, 2008.

22. D'Amelio P, Roato I, D'Amico L, et al: Bone and bone marrow pro-osteoclastogenic cytokines are up-regulated in osteoporosis fragility fractures. Osteoporos Int 22: 2869-2877, 2011.

23. Roato I, Brunetti G, Gorassini E, et al: IL-7 up-regulates TNF-alpha-dependent osteoclastogenesis in patients affected by solid tumor. PLoS One 1: e124, 2006.

24. Roato I, Gorassini E, Buffoni L, et al: Spontaneous osteoclastogenesis is a predictive factor for bone metastases from non-small cell lung cancer. Lung Cancer 61: 109-116, 2008.

25. Colucci S, Brunetti G, Rizzi R, et al: T cells support osteoclastogenesis in an in vitro model derived from human multiple myeloma bone disease: the role of the OPG/TRAIL interaction. Blood 104: 3722-3730, 2004

26. Kudo O, Sabokbar A, Pocock A, Itonaga I, Fujikawa Y and Athanasou NA: Interleukin-6 and interleukin-11 support human osteoclast formation by a RANKL-independent mechanism. Bone 32: 1-7, 2003.

27. Howlett M, Chalinor HV, Buzzelli JN, et al: IL-11 is a parietal cell cytokine that induces atrophic gastritis. Gut 61: 1398-1409, 2012.

28. Necula LG, Chivu-Economescu M, Stanciulescu EL, et al: IL-6 and IL-11 as markers for tumor aggressiveness and prognosis in gastric adenocarcinoma patients without mutations in Gp130 subunits. J Gastrointestin Liver Dis 21: 23-29, 2012.

29. Schinke T, Schilling AF, Baranowsky A, et al: Impaired gastric acidification negatively affects calcium homeostasis and bone mass. Nat Med 15: 674-681, 2009.

30. Juarez $P$ and Guise TA: TGF- $\beta$ in cancer and bone: implications for treatment of bone metastases. Bone 48: 23-29, 2011.

31. Maehara Y, Kakeji Y, Kabashima A, et al: Role of transforming growth factor-beta 1 in invasion and metastasis in gastric carcinoma. J Clin Oncol 17: 607-614, 1999.

32. Holen I and Shipman CM: Role of osteoprotegerin (OPG) in cancer. Clin Sci 110: 279-291, 2006.

33. Ito R, Nakayama H, Yoshida $\mathrm{K}$, et al: Expression of osteoprotegerin correlates with aggressiveness and poor prognosis of gastric carcinoma. Virchows Arch 443: 146-151, 2003.

34. Macsai CE, Foster BK and Xian CJ: Roles of Wnt signalling in bone growth, remodelling, skeletal disorders and fracture repair. J Cell Physiol 215: 578-587, 2008.

35. Glass DA II, Bialek P, Ahn JD, et al: Canonical Wnt signaling in differentiated osteoblasts controls osteoclast differentiation. Dev Cell 8: 751-764, 2005.

36. Morvan F, Boulukos K, Clement-Lacroix P, et al: Deletion of a single allele of the Dkk1 gene leads to an increase in bone formation and bone mass. J Bone Miner Res 21: 934-945, 2006.

37. Tian E, Zhan F, Walker R, et al: The role of the Wnt-signaling antagonist DKK1 in the development of osteolytic lesions in multiple myeloma. N Engl J Med 349: 2483-2494, 2003.

38. Li ZG, Yang J, Vazquez ES, et al: Low-density lipoprotein receptor-related protein 5 (LRP5) mediates the prostate cancerinduced formation of new bone. Oncogene 27: 596-603, 2008.

39. Voorzanger-Rousselot N, Goehrig D, Journe F, et al: Increased Dickkopf-1 expression in breast cancer bone metastases. Br J Cancer 97: 964-970, 2007.

40. Poon RT, Fan ST and Wong J: Clinical implications of circulating angiogenic factors in cancer patients. J Clin Oncol 19: $1207-1225,2001$

41. Fondevila C, Metges JP, Fuster J, et al: p53 and VEGF expression are independent predictors of tumour recurrence and survival following curative resection of gastric cancer. Br J Cancer 90: 206-215, 2004 